Ghislain POTRIQUET, Dominique HUCK et Claude TRUCHOT (sous la dir.) « Droits linguistiques » et "droit à la langue ». Identification d'un objet d'étude et construction d'une approche.

Limoges, Lambert-Lucas, 2016, 248 p

\title{
Asja Prohić
}

\section{OpenEdition}

\section{Journals}

Édition électronique

URL : http://journals.openedition.org/esp/1170

DOI : 10.4000/esp. 1170

ISSN : 2532-0319

Éditeur

Centre d'Information sur l'Éducation Bilingue et Plurilingue

Édition imprimée

Date de publication : 1 juin 2017

Pagination : 101-107

ISSN : 1127-266X

Référence électronique

Asja Prohić, «Ghislain POTRIQUET, Dominique HUCK et Claude TRUCHOT (sous la dir.) « Droits

linguistiques » et « droit à la langue ». Identification d'un objet d'étude et construction d'une approche

», Education et sociétés plurilingues [En ligne], 42 | 2017, mis en ligne le 01 février 2018, consulté le 23 septembre 2020. URL : http://journals.openedition.org/esp/1170 ; DOI : https://doi.org/10.4000/esp. 1170 


\section{COMPTE-RENDU d'OUVRAGE}

Ghislain POTRIQUET, Dominique HUGK et Glaude TRUGHOT (sous la dir.) "Droits linguistiques" et "droit à la langue». Identification d'un objet d'étude et construction d'une approche. Limoges, Lambert-Lucas, 2016, 248 p.

\section{Asja PROHIĆ}

A l'encontre de ce qu'ils qualifient de «doxa des droits linguistiques fondamentaux», attribuant aux droits linguistiques la qualité de droits de l'homme, les auteurs de ces actes du colloque tenu à Strasbourg les 25 et 26 septembre 2014 proposent une réflexion qui part de l'hypothèse que ces droits n'existent pas invariablement en tant que tels et qu'ils n'émergent que sous certaines conditions.

Afin d'appréhender ces conditions, ils adoptent une approche historique admettant volontiers les apports d'autres sciences humaines. Les phénomènes sont donc analysés de façons différentes, selon les spécialités géographiques et disciplinaires de chacun(e), proposant un large éventail de contextes à l'intérieur desquels peuvent être repérés les choix sociaux en matière de droits linguistiques.

L'ouvrage est divisé en deux parties, la première mettant l'accent sur les modalités d'émergence, et la deuxième sur les modalités de négociation des droits linguistiques.

Il s'ouvre sur la contribution d'O. Schneider-Mizony qui nous initie à la situation des immigrants d'origine germanophone au Brésil dans les années 1930-1940 qui se retrouvent confrontés à des restrictions de plus en plus importantes concernant l'utilisation de l'allemand dans l'espace social et privé. L'auteure interroge les divers facteurs qui ont conduit cette population forte d'environ 700000 personnes à la soumission et à l'obéissance teintées d'une expression strictement individuelle de mécontentement et au ressentiment: son hétérogénéité et isolement dans les zones rurales, sentiment d'illégitimité par rapport au Hochdeutsch, politique intégrationniste et totalitaire du Brésil de l'époque.

C'est encore des droits des germanophones dont il s'agit dans l'article suivant signé G. Potriquet, mais cette fois-ci aux EtatsUnis, pays, comme le rappelle l'auteur, aux deux constantes: un contact des langues conduisant à d'innombrables phénomènes de 


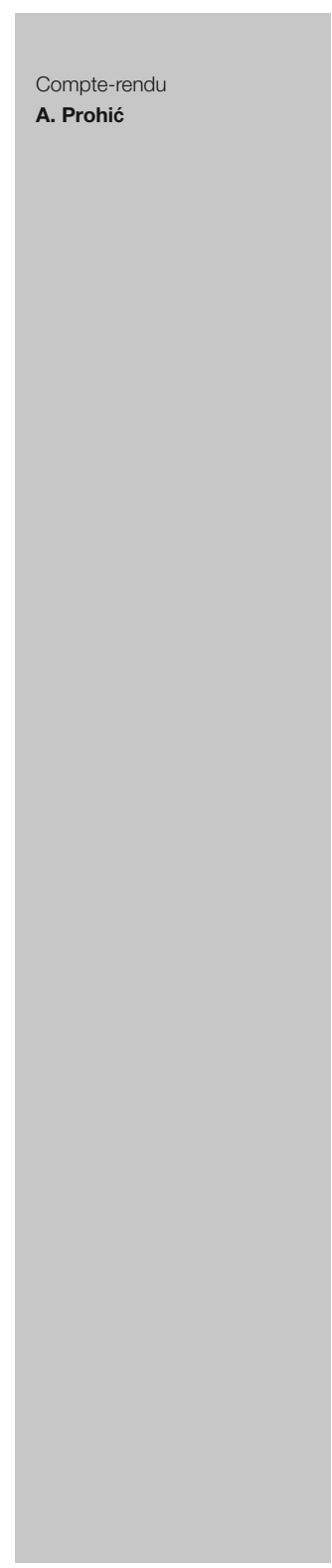

créolisation et de vernacularisation et une primauté de l'anglais s'étant imposé comme langue nationale sans aucune intervention délibérée du législateur. En prenant pour exemple deux affaires juridiques opposant d'un côté la communauté germanophone du Wisconsin de la fin du 19 ème siècle et celle du Nebraska au lendemain de la Première guerre mondiale respectivement à la loi Bennett et à la loi Siman, G. Potriquet met en lumière la manière dont les citoyens américains d'origine germanique ont su défendre leurs droits linguistiques. En s'appuyant sur la constitution des Etats-Unis et notamment sur son quatorzième amendement et plutôt que de s'opposer aux principes fondateurs de la laïcité et du civisme, ils ont su, avec le concours d'avocats et de juges favorables à leur cause, défendre l'enseignement en allemand au nom de l'autorité parentale, droit d'enseigner et de scolariser leurs enfants.

J.C. Herraras nous invite à découvrir le contexte historique dans lequel ont apparu les droits linguistiques en Catalogne. L'accroissement de l'utilisation du catalan dans la sphère publique à partir du 19 ème siècle est intrinsèquement lié à la naissance du catalinisme politique et le restera tout au long du siècle suivant jusqu'à nos jours. De la Deuxième République (1931-1939) jusqu'à la constitution de 1978, en passant par le régime de la dictature franquiste, les droits linguistiques des catalanophones vont toujours de pair avec des revendications d'autonomie politique. La politique linguistique volontariste de la Catalogne a d'abord trouvé son expression dans la «loi de normalisation» de 1983 et plus encore dans la «loi de politique linguistique» de 1998. Approuvée avec une importante opposition, la réforme du Statut d'autonomie de 2006 a porté au nombre de quatorze le nombre d'articles de loi sur les questions linguistiques.

La terminologie qu'on y voit employée, de «langue propre», «particulière» et «emblématique», pose aujourd'hui la question des droits de ceux et celles qui ont le castillan comme langue première et qui sont, d'ailleurs, plus nombreux que les catalanophones en Catalogne même.

Un autre exemple de l'usage des droits linguistiques à des fins politiques est mis en évidence par G. Raimondi qui fournit, dans son travail sur la dialectologie italienne, une analyse «d'étalage de cautions scientifiques» utilisé par les représentants de la Ligue du Nord pour revendiquer l'officialisation de ce qu'ils appellent la langue padane. S'appuyant à la fois sur la préexistence d'une idée de culture padane, les travaux du linguiste Hull et le cli- 


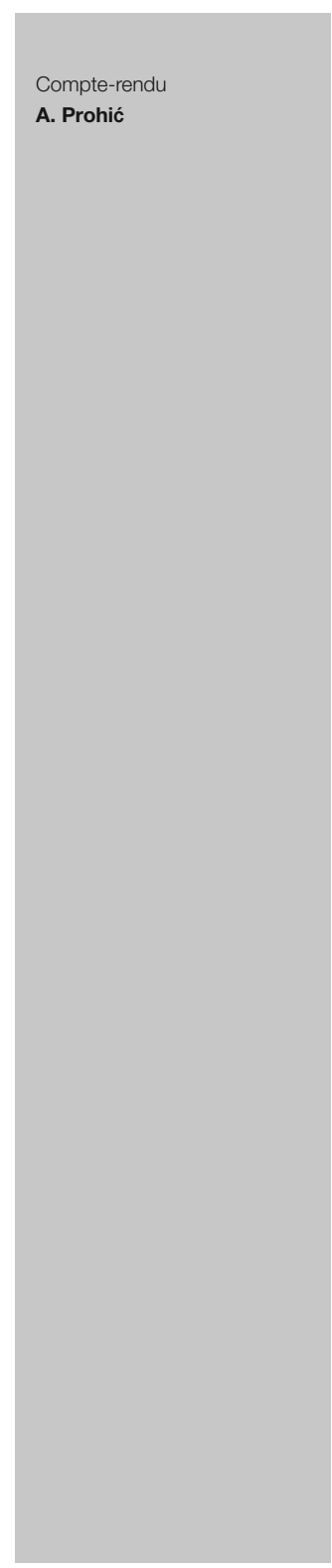

mat idéologique de valorisation des minorités, l'argumentation de la Ligue du Nord se construit graduellement à partir du constat que les dialectes de l'Italie du Nord sont similaires, pour conclure dans l'exigence de reconnaissance par l'Etat d'une langue-toit, la langue padane.

Cette revendication est, cependant, loin de faire l'unanimité des locuteurs qui, pour leur part, semblent préférer garder le caractère oral de leurs dialectes. Se pose ainsi la question, selon G. Raimondi, de la limite du concept de droit linguistique et les critères scientifiques et éthiques réglant son champ linguistique.

La première partie de l'ouvrage se clôt sur l'étude des discours publics en Alsace de la seconde moitié du 20 eme siècle. Elle est proposée par D. Huck. Sur la base d'une analyse des débats des sessions des Conseils Généraux du Bas et du Haut-Rhin entre 1945 et 1980, D. Huck nous interpelle sur la nécessité absolue de situer le discours et les différentes dénominations qui en découlent dans un ensemble de facteurs socio-historiques. En Alsace de l'après-guerre et compte tenu d'une politique linguistique radicalement tournée vers la diffusion du français et la limitation de l'usage de l'allemand et du dialecte alsacien, le droit linguistique ne se manifeste jamais en tant que tel et surtout pas dans une revendication quelconque. Il est plutôt question d'un «droit naturel» et de ce qu'on peut désigner comme une demande à exprimer les «possibilités linguistiques». L'objectif d'une telle stratégie argumentaire et énonciative est avant tout de faire comme si le français n'était pas concerné par cette demande. La logique développée est d'affirmer que dans un contexte historiquement sensible, les propos ne sont que d'ordre technique et purement fonctionnelle, permettant ainsi de contourner tout reproche de «politisation» des questions linguistiques. Ce n'est finalement qu'à partir des années 1980 et du changement de majorité politique en France que la question des langues en Alsace rebondira sous une nouvelle forme.

Le chapitre suivant consacré aux exemples de modalités de négociations des droits linguistiques s'ouvre sur la contribution de Y. Lefranc, interrogeant les divisions idéologiques autour du concept du Français Langue d'Intégration (FLI) à destination des migrants.

En effet, l'institution du FLI en 2011 conditionnant l'obtention de la nationalité française a suscité des débats, révélateurs des tensions sur des enjeux majeurs de la société française. S'appuyant en partie sur des témoignages des premiers concernés, formateurs en FLI et leurs stagiaires, Y. Lefranc nous guide à 


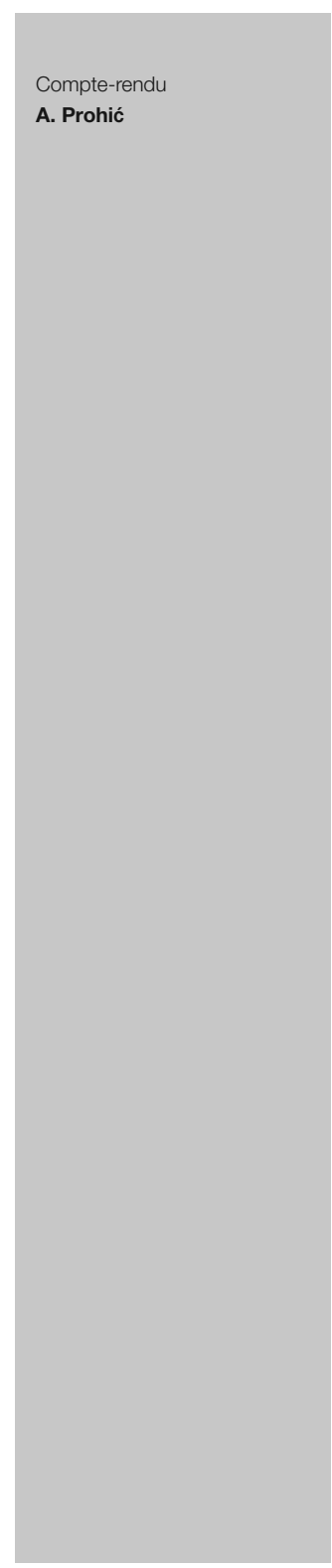

travers sa complexité en attribuant au FLI la force de «politiseur» de débat (la droite qui instaure, une partie de la gauche qui s'y oppose, les républicains sociaux qui rédigent son Référentiel, les associations qui la défendent, d'autres qui sont en désaccord avec ses principes...) et soulignant, pour sa part, une nécessité de rattacher la question au contexte des rapports sociaux de communication.

Il rend également manifeste, de par son analyse, la volonté d'enculturation des migrants par une surnormalisation des conduites (notamment à travers le volet civique du FLI) et pointe le doigt sur le caractère de dispositif allant ouvertement à l'encontre des travaux du Conseil de l'Europe qui prône le multiculturalisme et le régionalisme.

C'est justement des droits linguistiques au sein même des institutions européennes dont il est question dans l'article suivant signé J.-C. Barbier, spécialiste des politiques sociales de l'Union européenne. Alors que les publications de l'UE abondent d'éloges envers la diversité culturelle et linguistique de l'Europe et servent de base aux documents officiels formalisant une égalité parfaite entre les langues des pays la constituant, J.-C. Barbier démontre, à travers une analyse des pratiques propres aux institutions européennes et notamment leurs services juridiques, que l'anglais, hissé au statut de langue véhiculaire, y prédomine.

La principale conséquence de ce phénomène est que la majorité des citoyens européens qui ne maîtrisent pas l'anglais (70\%) sont de facto exclus d'une participation pleine et entière à la politique de l'Union. La confiance des citoyens dans l'UE est, par ailleurs, corrélés à leur niveau de formation et leur profession. Au lieu de les atténuer, l'UE participe ainsi à l'augmentation des inégalités entre les citoyens.

L'exemple suédois de «klarspråk», langue administrative claire et compréhensible, est aux antipodes de cette situation, et les facteurs historiques qui ont conditionné son avènement ainsi que sa base idéologique reposant sur la démocratie participative et délibérative qui façonne le modèle suédois depuis les années 1960, nous sont présentés par K. Ridell.

L'auteure décrit différentes étapes d'argumentation en faveur de «klarspråk» en s'appuyant sur une analyse du discours des textes officiels de 1960 à 2014 et fait le constat d'une volonté permanente de la part de l'Etat de répondre aux principes directeurs de la démocratie suédoise: transparence, efficacité et confiance. Plutôt que de donner des droits, il s'agit de remplir le devoir des autorités publiques envers ses citoyens en s'exprimant dans une 


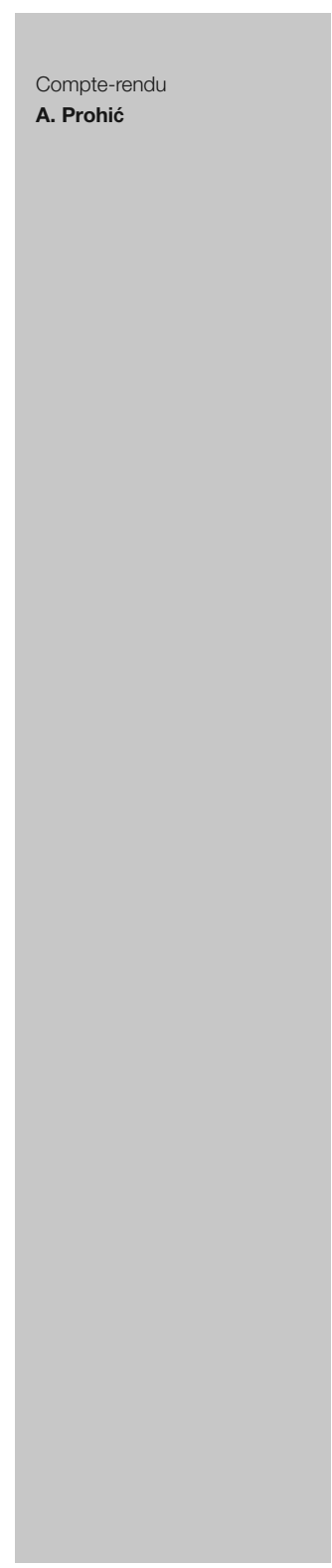

langue simple et facile à comprendre pour tout un chacun.

Dans sa contribution, G. Kremnitz interroge, à partir d'une typologie des politiques linguistiques dressée par lui-même quarante ans auparavant, les différentes possibilités que ces politiques offrent à l'expression du droit à la langue. Sa réflexion est complétée par une analyse de la présence de ce terme dans les encyclopédies des sciences du langage et les textes internationaux et l'évolution du concept en France.

Ainsi, la majeure partie de son travail porte sur la liste de langues que Bernard Cerquiglini, spécialiste de l'histoire de la langue française, avait élaborée en vue de la ratification par la France de la «Charte européenne des langues régionales et minoritaires». A partir de l'exemple de la liste Cerquiglini, G. Kremnitz pose les jalons d'une réflexion complexe sur le concept de la territorialisation des langues, concept totalement étranger à cette liste qui s'efforce de répondre à la demande tout en respectant les fondements de la République selon lesquels la France reconnait les droits de citoyens libres et égaux en droits et non de groupes disposant de droits spécifiques.

Le processus de négociation des droits linguistiques et les apports de la théorie libérale dans l'opposition droits individuels/droits collectifs est étudié, par la suite, dans le cas du Québec par L. Oakes.

En partant du fait que le français est la langue maternelle d'à peu près $80 \%$ de la population québécoise, mais de seulement $22 \%$ de la population de tout le Canada, l'auteur utilise les exemples de la langue de l'affichage public et de la langue d'enseignement pour exposer la manière dont le libéralisme multiculturel tel que défini par Will Kymlicka, philosophe politique, parvient à gérer ces droits concurrents.

Il s'agit de bien distinguer entre le choix de langue collectif et un choix de langue individuel. Si, aujourd'hui, tout citoyen du Québec a une obligation de respecter le choix collectif qui consiste à maintenir le français comme langue commune du pays, ce choix peut encore changer dans la mesure où il repose principalement sur une adhésion des Québécois à ce choix «social».

L'article suivant, proposée par S. Akin de l'Université de Rouen, examine les droits linguistiques des Kurdes en Turquie du point de vue historique et sociolinguistique.

Après des siècles d'un laissez-faire au sujet des langues parlées sur le territoire de l'Empire ottoman, la politique linguistique du nouvel Etat turc commence sa formation avec la constitution de 


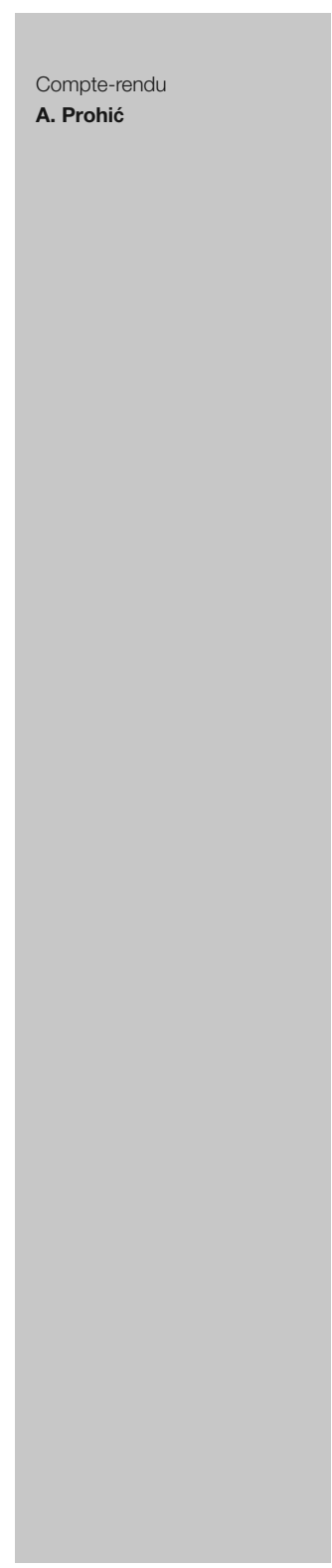

1924 qui déclare le turc langue officielle et devient, au fil des décennies, particulièrement répressive envers les locuteurs du kurde, atteignant son point culminant dans les années 1980. C'est, en effet, en 1983 qu'une loi interdit complétement l'usage d'autres langues que le turc (et donc avant tout du kurde comme deuxième langue parlée du pays) aussi bien dans le domaine public que privé (... il est interdit d'utiliser comme langue maternelle d'autres langues que le turc...).

A partir des années 2000, avec l'arrivée au pouvoir du gouvernement de l'AKP, et sans qu'elles permettent d'accorder un quelconque statut au kurde, plusieurs mesures sont prises en faveur notamment de la possibilité d'attribuer des prénoms non-turcs aux enfants ou de bénéficier d'un enseignement privé du kurde. Cependant, les dernières années, la tendance est à nouveau à l'opposé d'une plus grande reconnaissance.

Nous avons affaire ici, comme le souligne S. Akin, à des droits linguistiques intrinsèquement liés à la reconnaissance officielle de la population kurde en Turquie.

La dernière étude de cas nous vient de $\mathrm{K}$. Abiyeva qui analyse l'aménagement linguistique dans le Kazakhstan post-soviétique et fournit, à ce sujet, de précieux éléments nous permettant de mieux comprendre ses enjeux.

La langue russe, qui, durant la période soviétique, avait le statut de langue officielle utilisée dans les domaines administratif, juridique et législatif, et la langue dans laquelle ont été éduquées et scolarisées plusieurs générations de Kazakhs, reste, encore aujourd'hui, le principal référent socio-culturel.

La politique nationale de «kazakhisation» qui s'est mise en marche dans les années 1990 ne suffit toujours pas à faire basculer la balance du côté de la langue kazakhe, d'autant plus que le clivage entre les deux langues se double par des enjeux de langues minoritaires tel l'ouzbek.

Enfin, en conclusion de la réflexion collective que nourrit chacune des contributions du présent ouvrage, $\mathrm{C}$. Truchot revient sur le sens même d'une approche historique dans l'étude des droits linguistiques. C'est une approche qui permet de montrer, en s'appuyant sur des exemples pris, que les facteurs déterminant le traitement de ces droits ne sont pas que politiques, mais qu'ils sont imbriqués dans un ensemble de conditions d'ordre économique et social.

Les normes internationales qui servent d'ancrage à la revendication d'un tel droit à la langue ou de tels droits linguistiques, faisant abstraction d'histoires nationales fort différentes, ne font 


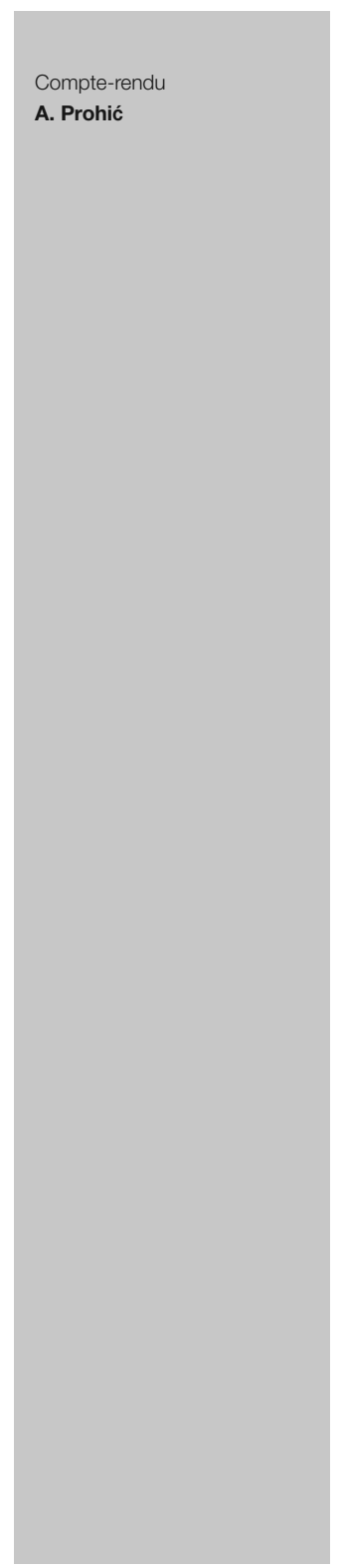

parfois que rendre ce traitement injuste.

C'est que ces droits ne doivent pas uniquement tenir compte de la complexité de l'être humain mais aussi de la complexité de leurs constructions collectives, les sociétés. En cela, nous ne pouvons pas nous passer de les reconsidérer dans un contexte historique. 